\title{
The effects of verbal labeling on short-term and incidental memory: A cross-cultural and developmental study
}

\author{
DANIEL A. WAGNER \\ University of Michigan, Ann Arbor, Michigan 48104
}

\begin{abstract}
Effects of induced verbal labeling on short-term and incidental memory were studied in Yucatan, Mexico. The 208 subjects, evenly divided by sex, were selected from four age groups $(7-8,10-11,13 \cdot 15$, 20-21 years) from a large public school. Stimuli were cards depicting both animals and objects familiar to all subjects, and were presented over 14 trials. Short-term memory was tested with a probed serial recall task on each trial; incidental memory was tested following the 14 trials. Several results conformed to findings with earlier studies using American subjects: short-term memory improved with age; primacy and recency recall were influenced by both age and labeling; and the typical inverted U-shaped incidental memory function was found. Verbal labeling apparently aided recall by focusing attention on the relevant items, but such overt labeling also impeded the strategy of verbal rehearsal used by oider subjects. Cultural factors appeared to play a limited role in the present study; the common element of formal schooling, among both American and Yucatecan subjects, was hypothesized as a possible explanation of such cross-cultural similarities.
\end{abstract}

Research on the effects of verbal mediation on certain cognitive tasks has stimulated considerable interest over the last dozen years (cf. Reese, 1962). In the discussion of short-term memory, attention has centered on the acquisition of task-appropriate strategies with increasing age (Belmont \& Butterfield, 1971; Flavell, 1970; and Hagen, 1972). Supplying verbal labels or "mediators" to children at certain ages appears to result in better recall and in changes in the serial position curves on short-term memory tasks (Bernbach, 1967; Hagen, Meacham, \& Mesibov, 1970). Specifically, it has been shown that overt verbal labeling leads to higher recall for children between ages 6 to 10 years, but does not increase recall for older subjects; these changes in recall are apparently due to increased recency recall for younger subjects, while older subjects show at the same time decreased primacy recall (Hagen, 1972). The development of incidental memory, studied in a wide variety of tasks, has been characterized by increased recall up to ages 12-14 years, followed by a decline-producing an inverted U-shaped function with age (e.g., Maccoby \& Hagen, 1965; Siegel \& Stevenson, 1966).

The number of cross-cultural studies of cognitive development has increased greatly in the last several years. These studies have provided evidence for both similarities and differences in memory development

This research was supported in part by a predoctoral traineeship (NICHD number HD 00149-06) granted to the author, and by a grant to Michael Cole of Rockefeller University (OEG-0-71-1965). The author wishes to thank Dr. Cole and Francisco Ix Can for their considerable help during the research. The author is also indebted to Harold $W$. Stevenson, John $W$. Hagen, and Robert V. Kail, Jr.. for critical comments. Finally, the cooperation of the principals, faculty, and students at Centro Escolar (Felipe Carrero Puerto) in Merida, Yucatan, Mexico is gratefully acknowledged. The author's address is: Department of Psychology, University of Michigan, Ann Arbor, Michigan 48104. across cultures, with formal schooling often considered to be an important factor (e.g., Cole, Gay, Glick, \& Sharp, 1971; Wagner, 1974). The general purpose of the present study was to determine if schooled Yucatecan children would show comparable memory development with American children. Specifically, the study was concerned on the effects of verbal labeling on age-related changes in short-term memory and the curvilinear trend in incidental memory.

\section{METHOD}

\section{Subjects}

The subjects were 208 males and females selected from a public school in Merida (Yucatan), Mexico. In the control condition, 16 males and 16 females were selected 'nonsystematically from the following four age groups: 7-8 (grade 2); 10-11 (grade 5); 13-15 (grade 8); and 20-21 (grade 12). The label condition consisted of 10 males and 10 females selected from each of the age groups listed above. In terms of socio-economic class, the subjects were from a broad middle range of family backgrounds, that included neither the very wealthy nor the very poor. They were primarily mestizo and generally spoke Spanish at home as well as in school; some subjects were bilingual and also spoke a Mayan dialect at home.

\section{Stimuli}

The task, adapted from Hagen et al. (1970), measured two cognitive processes: intentional short-term memory ("central" task); and incidental memory ("incidental" task).

In both the label and control conditions of the central task, the subjects were presented a series of seven cards, one at a time. Each card bore two colored pictures (an animal and an object). The pairs of pictures were as follows: fish-boot, frog-pitcher, bird-ladder, spider-ball, shrimp-bottle, scorpion-flower pot. The stimuli were drawn from a popular Mexican game, lotteria, and were recognizable to all the subjects, as shown by pretesting.

For the incidental task, two large cards were used: one contained all the animals, one the objects. When animals, (or objects) were the central task stimuli, the large card with all the objects (or animals) was used to test incidental recall. 


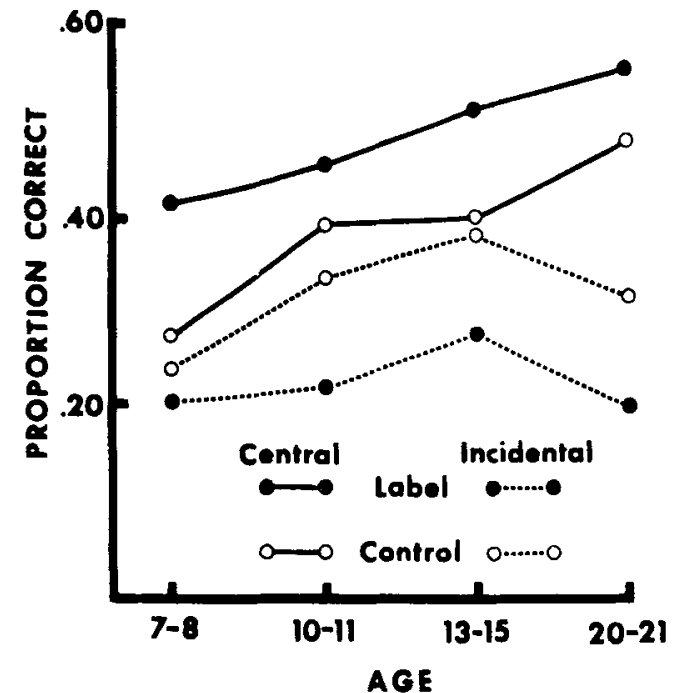

Figure 1. Central and incidental task performance by age and condition.

\section{Procedure}

The subjects were taken one at a time from the classroom to the testing room. Each card was exposed to the subjects for approximately $2 \mathrm{sec}$, and then placed face-down in front of the subjects, forming a row. Half the subjects were instructed to pay attention just to the animals, and the other half to the objects-these were the central (intentional) stimuli. In each case, the subject was told that he had to remember where in the row each of the animals (or objects) was located. A probe card which contained a single duplicate picture of one of the central stimuli, was presented about $2 \mathrm{sec}$ following the presentation of the series of seven cards. The subject was told to point to the card in the row where he thought the matching animal (or object) was located. The positions of the stimulus cards were randomized for all 14 test trials, with each serial position tested twice.

The central task was identical in both the control and label conditions, with one exception. In the label condition, as each card was presented, the subject was required to label overtly or "name" the central stimulus (i.e., the animal or object, depending on which set was central). Central task performance was the number of correct responses out of 14 trials.

The incidental task followed the central task. The large card containing the seven non-central (incidental) stimuli was presented to the subject. The subject was then presented, one by one, each of the central probe stimuli. For each probe picture, the subject was asked to point to the incidental picture that "always went with" that probe picture in the series. That is, the subject was required to recall as many of the animal-object pairs as he could. Incidental task performance was the number of correct pairings out of seven possible pairs.

All subjects were tested individually in an unused room of the school by a male experimenter. The experimenter was a Yucatecan of Mayan Indian extraction; he was bilingual in Spanish and Mayan. As all subjects spoke fluent Spanish, Spanish was used throughout the experiment. To maintain interest, the subjects were given candy or small amounts of money, with the amount determined by the age of the subject and contingent on the subject's performance. More details on task instructions and cultural setting are available in Wagner (1974).

\section{RESULTS}

\section{Central Task}

A three-way unweighted means analysis of variance, Condition (2) by Age (4) by Serial Position (7), with repeated measures on one factor (serial position) and unequal cells, revealed all main effects to be significant: condition, $[\mathrm{F}(1 / 200)=3.94, \mathrm{p}<.01]$, age $[F(3 / 200)=15.80, p<.01]$, and serial position, $[F(6 / 1200)=47.38, p<.01]$. The triple interaction was not significant.

The Condition by Age interaction, shown in Figure 1, was not significant. Labeling apparently increases short-term memory recall by a relatively constant factor at all ages. The Serial Position by Age interaction, shown in Figure 2, was significant $[F(18 / 1200)=3.41$, $\mathrm{p}<.01]$. It is apparent that the primacy portion of the serial position curve showed a considerable heightening with age, while the recency portion remained relatively unchanged.

The Condition by Serial Position interaction was also significant, $[F(6 / 1200)=13.21, p<.01]$, suggesting that verbal labeling has differential effects depending on the serial position of the item to be recalled. A further breakdown on this interaction, by primacy (Position 1) and recency (Position 7) is depicted in Figure 3. Separate analyses performed on primacy and recency recall indicated only two significant main effects: primacy recall increased with age, $[F(3 / 200)=12.86$, $\mathrm{p}<.01]$, and recency recall decreased in the label condition $[F(1 / 200)=72.21, p<.01]$. There were no other signiticant main effects or interactions. As mentioned earlier, studies with American school children

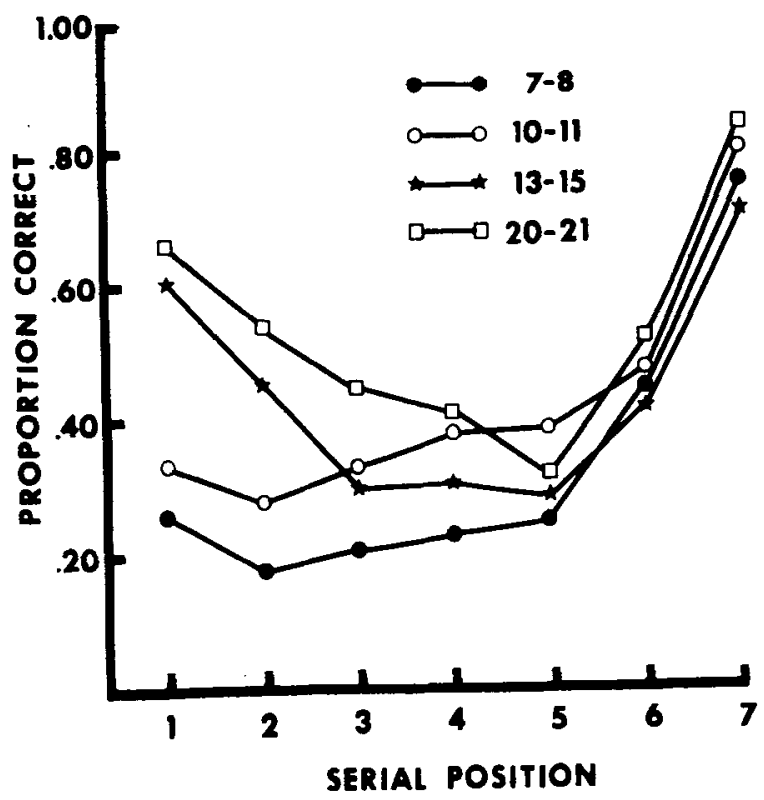

Figure 2. Central task performance by age and serial position. 
have shown that verbal labeling may cause a decrement in primacy recall for subjects over 10 years old. In the present study, primacy performance for older subjects (ages 13-15 and 20-21, pooled) was significantly poorer in the label condition in the expected direction $[t(102)=1.65, \mathrm{p}<.05]$.

A separate analysis indicated no significant differences that were attributable to sex.

\section{Incidental Task}

Incidental task performance, presented in Figure 1, indicated that verbal labeling produced a significant decrement in incidental memory recall $[F(1 / 200)=9.10, p<.01]$, while no other effects were significant. As in previous studies, the function of incidental memory appears to be curvilinear with age. In both conditions, recall increased up to age group 13-15, and then declined. A test of this decline was significant only in the control condition $[\mathrm{t}(62)=2.25, \mathrm{p}<.05]$.

\section{DISCUSSION}

The results of the present study replicate several previous findings: (1) verbal labeling produced increased recency recall over a wide age range; (2) verbal labeling has a detrimental effect on primacy recall for older children (over 10-11 years); (3) incidental memory recall increases until middle childhood, and then declines, producing the characteristic inverted U-shaped function.

Contrary to most findings with American subjects (e.g., Hagen et al., 1970) verbal labeling produced differences in overall performance on both the central and incidental tasks. The large augmentation in the recency recall, due to labeling, increased central recall over all ages, despite a decline in the primacy recall with older subjects.Furthermore, verbal labeling produced a decrease in incidental recall at all ages. Such a decrease is reasonable if one assumes that labeling has the effect of focusing attention on the central task stimuli. However, some investigators have stated that verbal labeling may serve as a "distractor" in that it tends to impair primacy recall (by interfering with verbal rehearsal), and in general seems to make selective attention to the central items more difficult (Hagen \& Hale, 1972). The present study supports only part of the above assertion. Indeed, verbal labeling seems to impair primacy recall for older subjects; but, for all subjects, such overt verbal labeling serves as an "attractor" by focusing attention on the central task stimuli. The data of this and one other study in which younger subjects were used (Wheeler \& Dusek, 1973) indicate that labeling produces an increase in central task recall and a decrease in incidental task recall. These findings are congruent with a large literature concerning the effectiveness of verbal mediators in directing the attention of children in certain cognitive tasks (as reviewed in Stevenson, 1972).

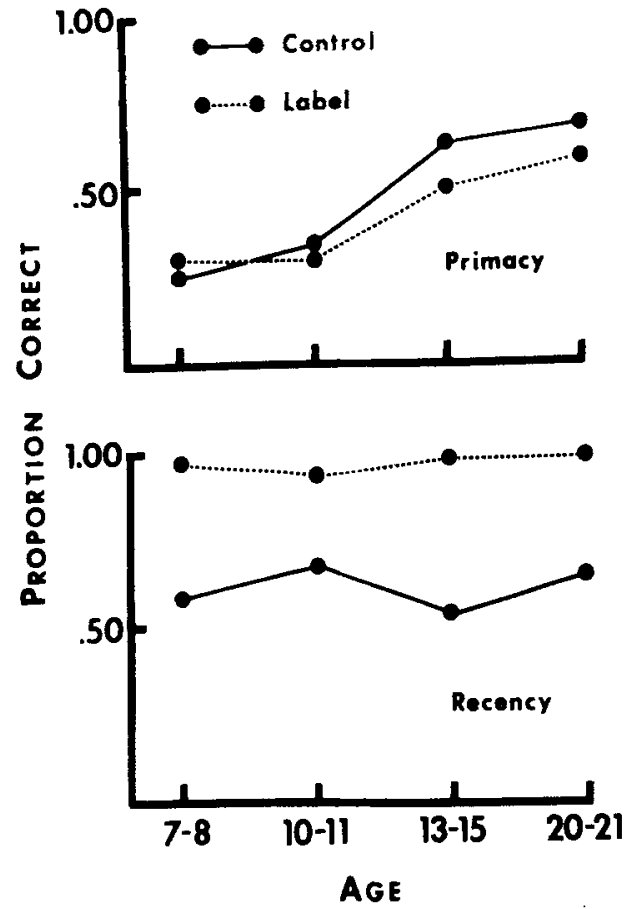

Figure 3. Primacy (Position 1) and recency (Position 7) recall in the central task by age and condition.

Cultural factors appeared to play a limited role in the present study. This may be primarily due to the fact that Yucatecan subjects were in school, as were American subjects in earlier studies. Schooling seems to be a "leveling" or equalizing factor that overshadows the possible effects of at least some cultural differences (Wagner, 1974). Nevertheless, some aspects of the present study, such as increased central task recall in the label condition, might be affected by certain cultural factors. In previous studies using American subjects, labeling produced increased recency recall and decreased primacy recall. As a result, total central task recall remained unchanged. In Yucatan, the gain in recency recall was considerably greater than the decrease in primacy recall. Thus there was a net increase in central task recall. At present, it is unclear whether such differences are produced by simple experimental variance, or by more complex cultural factors (such as differences in pedagogy, socialization, nutrition, mass media, et al).

In conclusion, these data tend to confirm, over a broad developmental age range and in a different cultural setting, the findings of previous studies, using American school children, with respect to short-term and incidental memory development, and the effects of verbal labeling. The mediational advantage of verbal labeling, in serial recall tasks, appears to be due to the increase in recall of the most recently presented items, and the focusing of attention on central, as opposed to incidental, information processing. 


\section{REFERENCES}

Belmont, J. M., \&utterfield, E. C. What the development of short-term memory is. Human Development, 1971, 14 236-248.

Bernbech, H. A. The effects of labels on short-term memory for colors with nussery chool children. Psychonomic Science, $1967,7,149-150$.

Cole, M., Gay, J., Glick, J., \& Sharp, D. The cultural context of leaming and thinking. New York: Basic Books, 1971.

Flavell, J. H. Developmental studies of mediated memory. In H. W. Reese and L. P. Lipsett (Eds.), Aduances in child development and behavior. Vol. 5, New York: Academic Press, 1970. Pp. 182-211.

Hagen, J. W. Strategies for remembering. In S. Farnham-Diggory (Ed.), Information processing in children. New York: Academic Press, 1972. Pp. 65-79.

Hagen, J. W., Hale, G. A. The development of attention in children. Paper presented at the Minnesota Symposia on Child Psy chology at the University of Minnesota. October, 1972

Hagen, J. W., Meacham, J. A, \& Mesibov, G. Verbal labeling. rehearsal, and short-term memory. Cognitive Psycholoty, $1970,1,47-58$.
Maccoby, E. E., \& Hagen, J. W. Effects of distraction upon central versus incidental recall: developmental trends. Journal of Experimental Child Psy chology, 1965, 2, 280-289.

Reese, $H$. W Verbal mediation as a function of age level. Psychological Bulletin, 1962, 59, 502-509.

Siegel, A. W., \& Stevenson, H. W. Incidental learning: A developmental study. Child Development, 1966, 37, 811817.

Stevenson, H. W. Children's learning. New York: Appleton-Century-Crofts, 1972.

Wagner, D. A. The development of short-term and incidental memory: A cross-cultural study. Child Development. 1974, 45, 389-396

Wheeler, R. J., \& Dusek, J. B. The effects of attentional and cognitive factors on children's incidental learning. Child Development, 1973, 44, 253-258.

(Received September 27, 1974.

Revision received December 16, 1974.) 